\title{
A Blockchain Technology Evolution between Business Process Management (BPM) and Internet-of-Things (IoT)
}

\author{
Doaa Mohey El-Din M. Hussein, Mohamed Hamed N. Taha, Nour Eldeen M. Khalifa \\ Faculty of Computers and Information \\ Cairo University \\ Egypt
}

\begin{abstract}
A Blockchain is considered the main mechanism for Bitcoin concurrency. A Blockchain is known by a public ledger and public transactions stored in a chain. The properties of blockchain demonstrate in decentralization as distribution blocks, stability, anonymity, and auditing. Blockchain can enhance the results of network efficiency and improve the security of network. It also can be applied in several fields like financial and banking services, healthcare systems, and public services. However, the research is still opening at this point. It includes a big number of technical challenges which prevents the wide application of blockchain, for example, scalability problem, privacy leakage, etc. This paper shows a proposed comprehensive study of blockchain technology. It also examines the research efforts in blockchain. It presents a proposed blockchain lifecycle which refers to an evolution and a linked ring between business process management improvement and Internet-of-Things concepts. Then, this paper presents a practical proof of this relationship for smart city. It presents a new algorithm and a proposed blockchain framework for 38 blocks (which recognized as smart-houses). Finally, the future directions are well presented in blockchain field.
\end{abstract}

Keywords-Blockchain; bitcoin; business process; cryptography; decentralization; consensus; applications

\section{INTRODUCTION}

A business process refers to a collection of related tasks to achieve the delivery process about service or product. Business process management (BPM) is keen on the design, execution, monitoring, and improvement of business processes. Systems include main four processes: analysis, design, enactment, and execution of the used processes in companies to streamline and automate intra-organizational processes. BPM is interested in improving corporate performance through managing the business processes [1].

The blockchain is a type of business process management but it makes a revolution in the management of processes as a study in optimization execution [2]. Blockchain technology can be a solution to interoperability, trust, and transparency issues in divider networks or systems. At its core, blockchain is a distributed ledger of asset and transaction records.

The term blockchain includes two threads a network and a data structure. Blockchain has a big difference from distributed database in data integrity. No one can update or delete any record in blockchain business process that will be a benefit and secure in many fields as healthcare or hospitals profiles about patients.

Blockchain is considered one of data structure unit/system [3], a blockchain includes a linked list of blocks, each containing a set of transactions. The crypto-graphical is the main property of each block in the chain. The data structure is duplicated across a network of instruments. Each instrument carrying the full replica is known a full node.

Blockchain is also a network when it is authorized a combination of peer-to-peer networks, consensus-making, cryptography, and market techniques. Blockchain's name that came from the data structure (fact) which a chained list of blocks. This chain is spread as a peer-to-peer network, in which every node retains the final updated version of it. This is considered that the immutable of blockchain history is very usefully.

The blockchain does not require to any authority reverse a central mechanism. It relies on a distributed node for sharing the data on the network. A consensus is required to achieve it on the network for each participant. In Bitcoin concurrency application, there is a function to deduce a consensus that is called a Proof of Work function [4]. This strategy requires that any node wishing to add a block to the blockchain must complete a computationally expensive (but easily verifiable) puzzle first. In healthcare applications, a patient can give the proof for the access of his information.

The Internet of things (IoT) [5] is a physical network for any resources can be controlled remotely as machines, devices, home appliances, and other items embedded systems. Each provenance had to make a software, sensors, and connectivity which enables these things to connect and change data. The development of intelligent objects is considered a distributed and decentralized ledger technologies with smart contracts. The internet of things (IoT) has an effective role in management and disruption these business practices. The main concepts must be taken into this process are security and accountability. They impose several rules with government regulations as finical systems. That causes a reduced costs and risk, reduce time delays, improved quality and consistency.

By integrating IoT [6] with decentralized blockchain technology that combines smart contracts, BPM and BPO will 
enter a world where just about anything can be connected to communicate intelligently.

This paper shows the relationship between blockchain and business process and internet of things. This evolution of relationship deduces main challenges are faced when constructing blockchain. The rest of this paper is organized as follows: Section 2 shows the related works. Section 3 is a presentation of Blockchain-Based on BPM \& IoT. In Section 4, outlines of the Blockchain advantages. Section 5 highlights the Blockchain challenges. In Section 6, the outlines of a case study on the smart city. In Section 7, the outlines of discussion. Finally, Section 8 the conclusion and future research direction.

\section{RELATED WORK}

This section presents a summary of the essential aspects of blockchain technology [7] and discusses initial research efforts at the intersection of BPM and IoT.

\section{A. Business Process Management (BPM)}

BPM is a business philosophy about people, and how to work together, and the performance objectives in their process. Business process management has a sequenced workflow including automate, monitor and analysis [2]. There are several distributions of lifecycle as in the following that has analysis, design, execution, implementation, monitoring and adaption.

The BPM lifecycle [8] differs as the organization or application but no one can exclude the main process analysis, design, execution and implementation. This lifecycle enables to apply the management system.

This paper illustrates several works on BPM and lifecycle, conditions, rules and structures.

The authors [9] presented a system for healthcare workflow in two hospital environments. They created the analytical framework based on six theoretical propositions identified as having a major impact on the implementation of workflow technologies.

In [10], researchers examined BPMS and presented a help in conditional structures such as if, switch, and while. They made a comparison between implementation of common conditional structures. This comparison relied on their workflow management systems via case studies, as well as discusses capabilities of each system.

In [11], the authors leaded to a BPMS research study. They discussed some details about workflow-related concepts and their typologies, references of some BPMS and current research trends and hotspots.

\section{B. Blockchain-Technology}

The blockchain [12, 13, and 14] is an original invention that can't be denied known by the pseudonym, Satoshi Nakamoto. A blockchain technology generated the backbone of a new type of internet. Bitcoin is an original digital concurrency which is based on blockchain technology. Blockchain [15] relies on four attributes and concepts: distributed shared leger, cryptography, consensus, smart contracts.
Blockchain came from the fact of its chained list of blocks as data structure. This chain of blocks distributes over a peerto-peer network, in which every node maintains the latest version of it. Blocks can contain information about transactions. Blockchain, the technology implicit Bitcoin, is a kind of Distributed Ledger Technology that has been known as a "distributed, shared, encrypted database that serves as an irreversible and incorruptible repository of information" [16].

A block includes a header and the body. The header of each block contains:

- Block version: refers to the rules of validation based on a set of blocks.

- Parent block hash: contains a 256-bit hash rate.

- Merkle tree root hash: the hash value of all the transactions in the block.

- Timestamp: refers to the timestamp by seconds currently.

- nBits: includes the existing hashing target in a compact format.

- Nonce: a 4-byte field, which usually starts with 0 and increases for every hash calculation.

Fig. 1 illustrates the blockchain structure and its components [17].

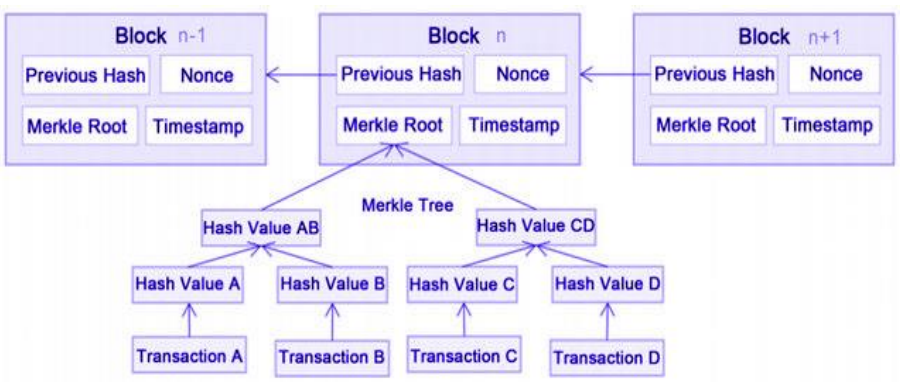

Fig. 1. The Blockchain Structure

The transaction counter and transactions are the two parts of the block body. The ultimate number of transactions for each block relies on the size of the block and the size of each transaction. A cryptography mechanism is used asymmetrically in blockchain to support and confirm the transactions' authentication [11]. The Digital cryptographic signature is utilized in an untrustworthy environment [12].

In the moment of the entered information, it could not be erased or updated. Blockchain is both the network and database in security and the data integration [13].

According to, the blockchain is a software communicator that can support making explicitly important architectural considerations on the resulting performance and quality attributes of the system. The authors' research [14] supported the architectural decision on whether to appoint a decentralized blockchain as opposed to other software solutions, such as traditional shared data storage. Additionally, they examined specific implications of the usage of blockchain as a software 
connector containing design trade-offs regarding quality attributes.

A semantic layer built upon a basic blockchain infrastructure would join the benefits of flexible resource/service discovery and validation by consensus. The researchers presented a novel Service-oriented Architecture (SOA) semantically [15].

The authors present BLOCKBENCH which is considered a benchmarking framework for performance perception of private blockchains versus data processing workloads. A study made a comprehensive evaluation of three major blockchain systems according to BLOCKBENCH [21] is entitled Ethereum, Parity and Hyperledger Fabric. The results are illustrated several trade-offs in the design space, as highperformance gaps between blockchain and database systems. Drawing from design principles of database systems, they were examined several research directions for bringing blockchain performance closer to the realm of databases [16].

\section{Business Processes Improvement by using Blockchain Technology}

Blockchain is like as a service that enables a business to leverage all the advantages of cloud computing while the blockchain assessment and implementation: flexibility, agility, capex-free, compliance, scalability, pay as you go, allowing business to deal with decentralized blockchain network concurrently. A time can be saved in creating blocks, managing a blockchain network, designing the network, and the developing applications, swiftly pool and validate use cases. Rapidly scale and roll out blockchain based services.

\section{1) Blockchain Levels}

There are three levels of Blockchain [17]: Storage for digital records, Exchanging digital assets, and Implementing the smart contracts requires knowing the basic rules, and understanding terms, properties and conditions recorded for the implemented code. Distributed network performs contract \& monitors compliance. The results are evaluated without the third party automatically.

\section{2) Blockchain Types}

The types of blockchain include three formative mechanisms as in Fig. 2: verify it.

a) Public: Everyone can check the transaction and

b) Consortium: It refers to the node that had authority can be chosen in advance, usually has partnerships like business to business, the data in blockchain can be open or private, can be seen as Partly Decentralized.as Hyperledger.

c) Private: it refers to not every node can participate, maybe one or more restricted in the constructed blockchain. That interpretation of the firm authority for the access of data management.

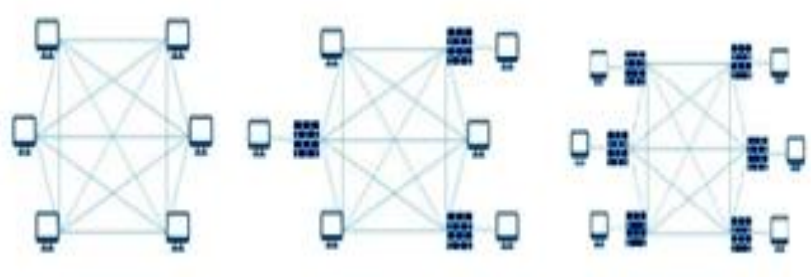

Fig. 2. Types of Blockchain

\section{3) Blockchain \& BPM Applications}

In addition, a private-public key mechanism coupled with powerful cryptographic algorithms keep everything secure. Blockchain applications damage conventional thinking and conventional ways according to the processing of data, handling, and storage.

The inter-organizational processes used blockchain represent [2]: the control flow as a big part in blockchain and business logic of its processes can be executed from the process models into the blockchain smart contracts. That is known trigger components allowed connecting these interorganizational process implementations to Web services and internal process implementations. These triggers can build a bridge between the enterprise applications and the technology of blockchain. The cryptocurrency basic can enable the selective implementation of conditional payment and built-in escrow management at defined points within the process, where this is required and feasible to clarify these capabilities. This may very well be a basis for misunderstandings and shifting blame in cases of conflict $[2,3]$.

The technical realization of this advance is still nascent at this stage, although some early efforts can be found in the literature. For example, smart contracts that implement the trust execution process from BPMN process models [2] and from domain-dependent [18]. Further, the evaluation of optimizations costs is presented by [3].

The previous examples presented that blockchain technology and how to apply on BPM application. It is important to pass the real technical issues blend with promising application scenarios; early implementations mix with unanticipated challenges.

\section{Blockchain in IoT and Blockchain Applications}

Internet of things (IoT) [5] is considered a worldwide network of interconnected objects and human beings, which through singular addressing schemes are able to interact with each other and participate with their neighbors to reach common targets [19].

The primary purpose of IoT is to share objects and entities information that examines the manufacture, transportation, and other specifics of people's life. Through the Iot information, it could produce a preferable cognitive and environment. But the development of the IoT is still slowly these years. IoT has a lifecycle for applying in any domain, as in the following: 
This lifecycle is faced several problems in blockchain technology. That may be affected on the time and confideability. Because when the blockchain applies that required approved from all blocks to any updates.

One important reason is that the high costs of the deployment. The security and privacy of Internet of Things (IoT) exist major challenge because of the big scale and distributed nature of IoT networks. The approaches of blockchain technology serve the decentralized security and support the privacy. So far, they comprise significant energy, delay, and overhead that is not appropriate for most resourceconstrained IoT devices.

The essential logistics basics are traceability and transparency. IBM Blockchain makes optimization business transactions and trading relationships with substantially secure business networks on blockchain - both at scale and globally.

Blockchain companies attracted \$ 525 million in 2015, largely as a result of a peak in investments in the first quarter of 2015. Investments then decreased until the fourth quarter of 2015 when only \$45 million was invested $67 \%$ of the fundraising activities carried out between January 1, 2015 and February 18, 2016 concern companies specializing in Blockchain infrastructure and applications. The remaining 33\% are companies specialized in bitcoin [20]. Fig. 3 illustrates the finical sectors percentage using blockchain technology.

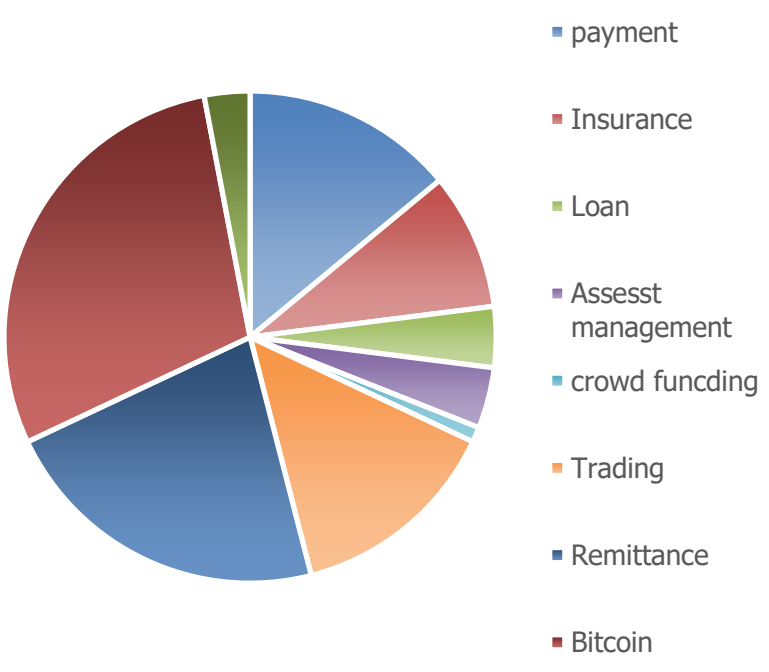

Fig. 3. Financial Sectors used Blockchain Distributions

This section discusses applications and examples for using blockchain in IoT.

1) Electric Power Microgrids:

Electric power can be used blockchain in different block sizes as big or small. Smart contracts are being used for redistributing excess power from solar panels. The Transactive Grid is an application running on blockchain to monitor and redistribute energy in a neighborhood microgrid. The program presented saving costs and reducing pollution through buy and sell processes automatically. The technology for running the program is the Ethereum platform, designed for building smart contracts of any kind [21].

\section{2) Cold Chain Monitoring}

Food and pharmaceutical products mostly want a specific pilling. Also, enterprises also see the value in sharing warehouses and distribution centers, instead of each one paying for its own. Sensors on sensitive products can record temperature, humidity, vibration, and other items of interest.

These readings can then be stored on blockchain. They are permanent and tamperproof. If a storage condition deviates from what has been agreed, each member of the blockchain will see it. A smart contract can trigger an action to correct the situation. Depending on the size of the deviation, this action may be to simply adjust the storage. However, it could also extend to changing "use-by" dates, declaring products unfit, or applying penalties [22].

\section{3) Meat Traceability}

Product status at each stage of production can be recorded using blockchain. The records are permanent and inalterable. They also allow the tracing of each product to its source. Global retailer Walmart uses blockchain to track sales of pork meat in China. Its system lets the company see where each piece of meat comes from, its processing and storage, and sellby date. In the event of product recall, the company can also see which batches are concerned and who bought them [23].

\section{4) Automotive Supplier Payments}

Blockchain allows the transfer of funds anywhere in the world. Bitcoin transfers specifically also offer lower fees. Australian vehicle manufacturer Tomcar uses bitcoin to pay suppliers. The advantage is in the cost savings. On the other hand, the firm is careful to avoid hanging onto too much bitcoin. While bitcoin is international by nature, some national governments see it as a way for companies to make an investment. Companies with bitcoin holdings may, therefore, be taxed accordingly [24].

They presented [25] a lightweight instantiation of a BC particularly geared for use in IoT by eliminating the Proof of Work (POW) and the concept of coins. The authors' research was exemplified in a smart home setting and consists of three main tiers namely: cloud storage, overlay, and smart home. They presented that Blockchain smart home system is secure by thoroughly analyzing its security. They introduced results of their simulation and the overheads.

\section{COMPERHENSIVE STUDY}

This paper presents a comprehensive study between ten research papers about blockchain related to business process management (BPM) and Internet-of -things (IoT) as in Table I.

\section{BLOCKCHAIN-BASED IN BPM AND IOT}

There are also challenges and opportunities for BPM and blockchain technology beyond the classical BPM lifecycle. We refer to the BPM strengths [26] beyond the methodological support we reflected above, including strategy, governance, information technology, people, and culture. 
TABLE I. The COMPREHENSIVE STUdy BETWEEN TEN RESEARCHES RELATED TO BLOCKCHAIN AND BusinESS PROCESS

\begin{tabular}{|c|c|c|c|c|}
\hline Paper No. & BP challenge & Technique used & Domain & Goal \\
\hline$[4]$ & $\begin{array}{l}\text { Risk of centralized data is the security of } \\
\text { taking footprint and requires centralized } \\
\text { trust in a single authority }\end{array}$ & $\begin{array}{l}\text { Block chain in three } \\
\text { algorithms: } \\
\text { Creating blocks } \\
\text { Proof of interoperability } \\
\text { Miner election. }\end{array}$ & Health & $\begin{array}{l}\text { Describe an approach to } \\
\text { effectively and securely share } \\
\text { healthcare information } \\
\text { within a data sharing network }\end{array}$ \\
\hline [26] & Risk Adaptations & $\begin{array}{l}\text { The companies Study } \\
\text { works on blockchain } \\
\text { technology based }\end{array}$ & Financial & $\begin{array}{l}\text { Study improvement of blockchain } \\
\text { in business as Bitcoin } \\
\text { concurrency efficiency as Visa, } \\
\text { Mastercard, Banks, NASDAQ, } \\
\text { etc., are investing in exploring } \\
\text { application of current business } \\
\text { models on Blockchain. }\end{array}$ \\
\hline$[27]$ & $\begin{array}{l}\text {-T capabilities and Infrastructure } \\
\text {-Information and cyber security } \\
\text {-Integration and collaboration }\end{array}$ & Supply chain & Delphi study's expert & $\begin{array}{l}\text { portraying the emerging transition } \\
\text { trend from a digital business } \\
\text { environment, the presented } \\
\text { Delphi study findings contribute } \\
\text { to extant knowledge by } \\
\text { identifying } 43 \text { opportunities and } \\
\text { challenges linked } \\
\text { to the emergence of Big Data } \\
\text { Analytics from a corporate and } \\
\text { supply chain perspective }\end{array}$ \\
\hline$[13]$ & $\begin{array}{l}\text { Scalability problems: } \\
\text { storage optimization of blockchain } \\
\text { re-designing blockchain }\end{array}$ & $\begin{array}{l}\text { Blockchain testing could } \\
\text { be separated into two } \\
\text { phases: standardization } \\
\text { phase and testing } \\
\text { phase }\end{array}$ & Organization & $\begin{array}{l}\text { They presented a } \\
\text { Comprehensive survey on } \\
\text { blockchain including blockchain } \\
\text { architecture and key } \\
\text { characteristics of blockchain. }\end{array}$ \\
\hline$[21]$ & $\begin{array}{l}\text { Performance evaluation, bridging } \\
\text { Database Design into data model layer for } \\
\text { BC, and Scalability }\end{array}$ & BlockBench & YCSB small bank & $\begin{array}{l}\text { BlockBench measures overall and } \\
\text { component-wise performance } \\
\text { regarding throughput, latency, } \\
\text { scalability, and fault-tolerance }\end{array}$ \\
\hline$[16]$ & $\begin{array}{l}\text { the adoption of blockchain in the supply } \\
\text { chain and logistics }\end{array}$ & $\begin{array}{l}\text { Financial and non- } \\
\text { financial spheres }\end{array}$ & Industries & $\begin{array}{l}\text { Consider the possible adoption of } \\
\text { blockchain-based application, } \\
\text { created by the Finnish company } \\
\text { Kouvola Innovation. }\end{array}$ \\
\hline$[20]$ & $\begin{array}{l}\text { Performance evaluation for semantic- } \\
\text { enhanced blockchain }\end{array}$ & $\begin{array}{l}\text { Logistics, industry, Utility } \\
\text { markets, Public sector, } \\
\text { Financial services }\end{array}$ & $\begin{array}{l}\text { several domains of } \\
\text { smart cities and } \\
\text { communities }\end{array}$ & $\begin{array}{l}\text { Improve performance for } \\
\text { Semantic based blockchain } \\
\text { enhancement in different domain }\end{array}$ \\
\hline$[23]$ & $\begin{array}{l}\text { the unambiguous and correct specification } \\
\text { of smart contracts }\end{array}$ & $\begin{array}{l}\text { the structure of Nested } \\
\text { ADICO (nADICO) }\end{array}$ & $\begin{array}{l}\text { domain-specific } \\
\text { language }\end{array}$ & $\begin{array}{l}\text { It can automate the translation of } \\
\text { institutional constructs into } \\
\text { codified machine-readable } \\
\text { contractual rules. }\end{array}$ \\
\hline$[15]$ & $\begin{array}{l}\text { Scalability and performance of The main } \\
\text { bottlenecks in Hyperledger and Ethereum } \\
\text { are the consensus protocols }\end{array}$ & BLOCKBENCH & $\begin{array}{l}\text { a domain name } \\
\text { registrar }\end{array}$ & $\begin{array}{l}\text { Improving blockchain } \\
\text { performance }\end{array}$ \\
\hline$[6]$ & blockchain-IoT combination & $\begin{array}{l}\text { distributed peer-to-peer } \\
\text { systems }\end{array}$ & Several industries & $\begin{array}{l}\text { It makes blockchain-IoT } \\
\text { combination to facilitate the } \\
\text { sharing of services and resources }\end{array}$ \\
\hline
\end{tabular}


And the strengths [28], if we use blockchain in IoT, are low cost, Flexible system, higher Security, Systematic, and high efficiency. We find the new role to can combine between the two concepts of BPM and IoT, how to enter IoT in blockchain lifecycle according to manage a business process.

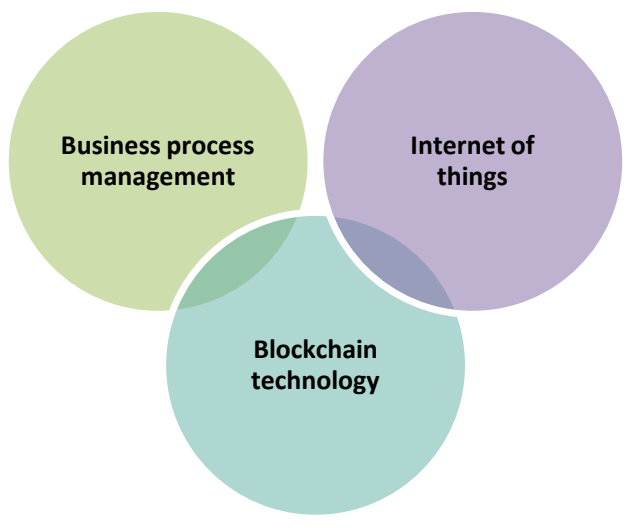

Fig. 4. The relationship between blockchain to BPM \& IoT.

This relationship will reflect on blockchain lifecycle and differs in the processes sequences or importance. Blockchain technology raises another relevant perspective for estimating high-level processes in terms of the implied strengths, weaknesses, opportunities, and threats.

The proposed lifecycle merges between the main characteristics and how to affect each process in the next process in business process management. As a result of the Fig. 4, IoT can serve in management systems and be a system high secure, that shows the relationship between blockchain to BPM and IoT. This lifecycle has several challenges as scalability, security, complexity, confidentiality, and domain dependent.

\section{A. The Blockchain Lifecycle}

The blockchain lifecycle includes 7 processes. These processes are:

1) The Analysis: is concerned to acquisition insights into issues relating to the way a business process currently operates. Each organization can apply the blockchain processes analysis shared internally and externally by stakeholders [28]

2) IoT security people: Blockchain Enforces rules BPMrelated information technology subsumes all systems that support process execution, such as process-aware information systems and business process management systems. These systems typically assume central control over the process. Blockchain technology might modify governance to be more externally depending on smart contracts [29].

3) Design [30, 31]: which refers to the identification and distribution of the blocks on the network. It also determines network type (private, public or hybrid models). And the security and authority for each block through the network.

Fig. 5 illustrates the proposed lifecycle of relationship in blockchain and BPM \& IoT 4.

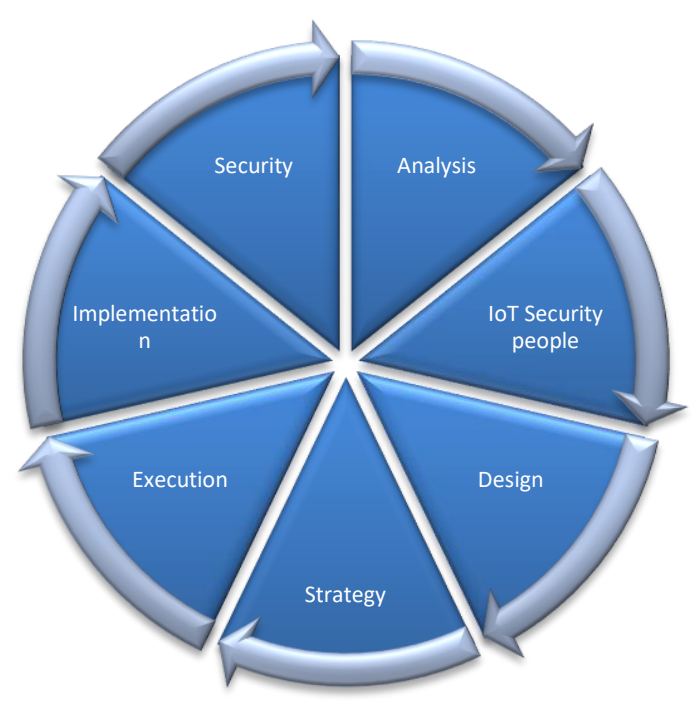

Fig. 5. The proposed lifecycle of relationship in blockchain and BPM \& IoT.

4) Execution: that is keen on the instantiation [32, 33] of individual cases and their information-technological processing. Recently, it is important to aware each process in the information systems and in the management systems [34]. The essential challenge of the implantation engineering level is the identification and definition of abstractions for the design of blockchain and processes execution.

5) Implementation: refers to the procedure of transforming a to-be model into software components executing the business process [34]. In this context, the question is how can the involved parties make sure that the implementation that they deploy on the blockchain supports their process as desired. Some of the challenges regarding the transformation of a process model to blockchain artifacts are discussed by [35].

6) Monitoring Process: is supportive events for implementing and executing way, and triggering alerts to identify strange behavior [36].

\section{B. Organizational knowledge}

Organizational knowledge is defined by the collective values of a group of people in an organization [13]. Currently, BPM is discussed in relation to organizational culture [37] from a perspective that emphasizes an affinity for clan and hierarchy culture.

Blockchain privacy and Governance refers to appropriate and transparent accountability in terms of roles, responsibilities, and decision processes for different BPMrelated programs, projects, and operations [3]. Currently, BPM can define the roles of BPM and properties in each organization internally. Blockchain technology provides a governance model orientation. Research on corporate governance investigates agency problems and mechanisms to provide effective incentives for intended behavior. Smart contracts can be used to establish new governance models as exemplified by The Decentralized Autonomous Organization (The DAO). 


\section{BlOCKCHAIN TECHNOLOGY ADVANTGES}

The blockchain can improve the security and transparency through all kinds of transactions, put the probabilistic of the possibilities. It also can represent at the supply chain [16]. This technology enhances in the tasks:

- Recording the quantity and transfer of assets - like pallets, trailers, containers, etc. - as the nodes movement of supply chain.

- Tracking purchase orders, change orders, receipts, shipment notifications, or other trade-related documents

- Assigning or verifying certifications or certain properties of physical products; for example determining if a food product is an organic or fair trade.

- Linking physical goods with digitalizing numbers or codes.

- Sharing information.

Blockchain offers shippers the following advantages:

- Improved Transparency. Documented products

- Greater Scalability. Virtually any number of participants, accessing from any number of touchpoints, is possible.

- Better Security. it can share the data in ledger to audit the requirements and support company internally.

- Increased Innovation. Opportunities abound to create new, specialized uses for the technology as a result of the decentralized architecture.

- Confidant-Ability

- Data integrity

\section{BLOCKCHAIN TECHNOLOGY CHALLNEGES}

There are several challenges $[38,39]$ in blockchain but this paper focuses on the challenges when blockchain mechanism intervenes in BPM and IoT concepts. The proposed lifecycle faces challenges when anyone tries to build it. These challenges are shown as scalability, security, complexity, speed cost and domain dependent.

1) Scalability Blockchains: This refers to the big size of blockchain network and grows continuously. That may cause of several attacks on this network. So that requires often distributed management systems and powerful network to harvest any problem on the network.

2) Trade-off Transaction Costs and Network Speed: According to several political aspects when using bitcoin, there is a problem of store information and how to get miners the information or rerecord them.

3) The blockchain Complexity: It has made cryptography more mainstream. The recent researches support several types of glossaries and searching indexes to make it easy to understand.
4) Confidentiality: Mute information in a circle and do not change it to protect the data.

5) Based on specific-Domain: Each blockchain relies on one structure of domain with basic rules and conditions of it. No one till now can enter several data about different topics to cover different topics in each block. That is a problem in management data and resources.

6) Security: The still open research problem insecurity illustrates in the lying or rumor review. If more than a number of half working persons on the network say one lying that will be a true fact.

For this reason, the mining of bitcoin pools are demonstrated carefully by the community, to guarantee no strange people on the network. That means the Politics in blockchain: the protocols of the blockchain present a chance to digitize governance models. Another reason, miners are ultimately forming another kind of incentivized governance model, there have been ample chances for public collisions between various community strips.

There are other challenges to deal with the blockchain technically:

1) Mindset: Blockchain was targeted for the decentralized research. There is still a problem in mind thinking in the idea of the centralization for the most students or researchers in blockchain orientation.

2) Human Error: If a blockchain is used as a database, the information going into the database needs to be of high quality. The blockchain stores data unstructured, so that requires to evidence when registering the data correctly in first computer/block.

3) Technology And Know-How: Blockchain programming takes a mix of software skills. It also helps to understand economies and businesses, especially your business. You may have to train staff or hire new people with these skills. The development of blockchain outsourcing that can support the third party.

\section{EXPERIMENT STUDY}

If executed using smart contracts on a proposed life cycle of blockchain, typical barriers complicating the deployment of smart-city.

- The blockchain supports a fixed public ledger so the entrants can trust the history writing messages to define the error source.

- Smart contracts can monitor the independent process globally. That interparty only predicated messages are agreeable.

- The data will be encrypted for visible in public blockchain network. These capabilities examine the useful blockchains for communities and organizations to perform the business and organizations' boundaries.

This is an essential improvement, because the blockchain core uses to provide enterprise collaborations going far beyond asset management, raise safety or sharing the personal smart 
city records in purchase (home) block providers as in the following, which we propose this algorithm.

In this experiment, we design small simulation architecture in smart cities. That targets higher security system for each owner/ node in the city and easier to sell and buy process from the trusted owners. Fig. 6 shows the proposed experiment of Blockchain in smart city.

Blockchain also can control automated systems for several owners/ users in each building. Blockchain technology relies on distributed decentralized ledgers. The simulation works on thirty-eight computers and virtual computers (distributed devices and databases) in a lab to test the blockchain concept.

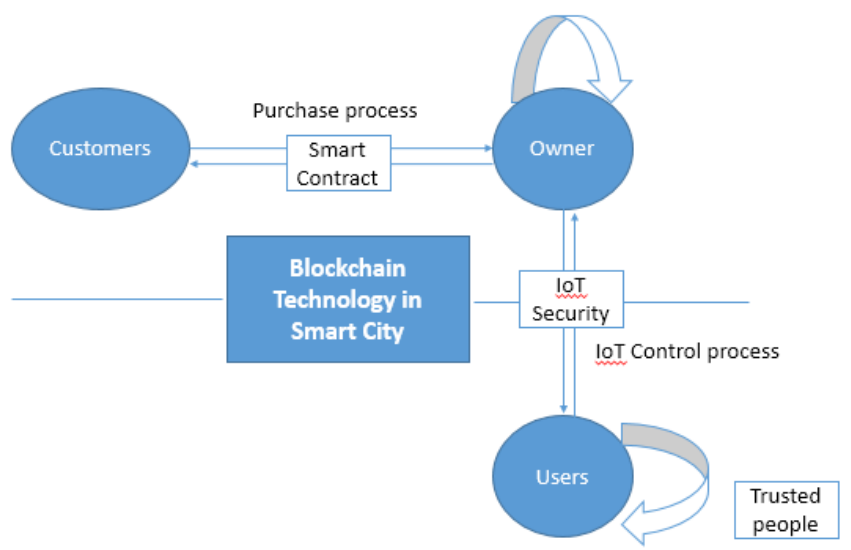

Fig. 6. Blockchain in Smart city

\section{1) Contract Signing Stage}

Contracts are signed by electronic data interchange (EDI) in traditional E-business [40], and digital signature is used to guarantee the legal effect. Other insurance measures include negotiation logs and files. This electronic evidence can be used to arbitrate the legal dispute in the transaction. But these evidences have to be kept in a server that is managed by a third party.

\section{2) Contract Fulfillment Stage}

This stage starts after both the buyer and seller have completed all the procedure of the contract. In the classical Ebusiness, the seller should be ready for customers' requirements in goods and issues by the evidence, insurance and available credit then the delivery process for goods with transporting an organization to finish rest tasks as packing, shipping and transportation. The buyer should transmit the money of goods through banks accounts after confirming buying process of these goods. Even if these smart contracts are preserved by some companies or organizations, there is no guarantee that the content of them will not be modified or delete by someone. Therefore, we need to publish the smart contract into the Blockchain. Here are two transaction styles in IoT E-business. One is the payment, the other is the exchange. The formal one can be applied to the purchase of the commodities and services on the IoT.

Table II presents the proposed Blockchain Algorithm in Smart City. In other words, this table can discuss the smart housing for Building City Internally. For example, one people want to buy commodities from a DAC. For example, B want to buy a car from A. First, Smart contract includes the exact terms of the transaction. Second, both sides confirm this contract and publish it into the Blockchain. Lastly, the contract will take effect and both sides will get what they need.

\section{TABLE II. The Proposed BlockChain Algorithm IN SMART City} (SMART HOUSING FOR BUILDING CITY INTERNALLY)

1. Function PurchaseBuilding (id)

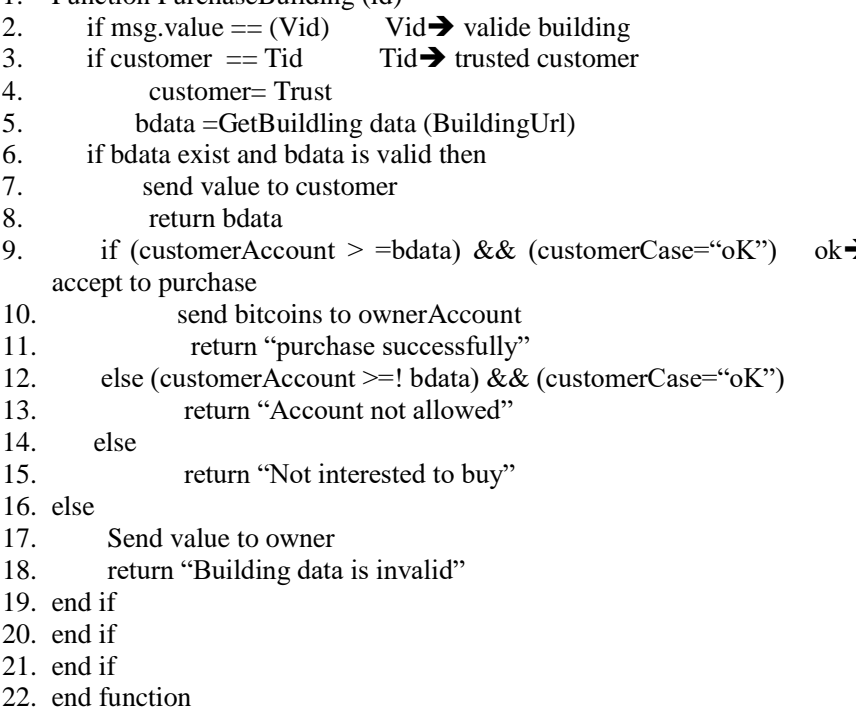

\section{CONCLUSION AND FUTURE WORK}

This paper presents a comprehensive study of blockchain technology and its effect in business process management and Internet-of-thing. It presents IoT life cycle and its relation between BPM lifecycle. It also proposes a solution for higher security in blockchain in a smart-city. Blockchain can transform supply chains, industries and ecosystems. Smart contracts can save time and cost and eliminate any delay. So that make the chain is faster and more intelligent and higher security of supply chain.

\section{REFERENCES}

[1] Book chapter: Introduction to Business Processes, BPM, and BPM Systems, Springer International Publishing Switzerland 2015 A. Burattin: Process Mining Techniques in Business Environments, LNBIP 207,

[2] Mendling, J., Weber, I., Van Der Aalst, W., Brocke, J. V., Cabanillas, C., Daniel, F., ... Zhu, L., Blockchains for Business Process Management - Challenges and Opportunities. ACM Transactions on Management Information Systems, 9(1), 2018.

[3] Luciano, G., Alexander, P., Marlon, D., and Ingo, W., Optimized execution of business processes on blockchain, BPM'17: International Conference on Business Process Management, Barcelona, Spain, 2017.

[4] Kevin, P., Rammohan, D., Pradip, K., and Kelly, B., Mayo C., A Blockchain-Based Approach to Health Information Exchange Networks, 2016

[5] Dave, E., The Internet of Things How the Next Evolution of the Internet Is Changing Everything, Cisco Internet Business Solutions Group (IBSG), 2011.

[6] Konstantinos, C., \& Michael, D., Blockchains and Smart Contracts for the Internet of Things, 2016 IEEE. Translations and content mining are permitted for academic research only, volume 4, 2016.

[7] Allan, T., and John, D., Linked Data Indexing of Distributed Ledgers, WWW '17 Companion Proceedings of the 26th International Conference on World Wide Web Companion, Australia 2017. 
[8] Stefan R. Koster, An evaluation method for Business Process Management products, University of Twente, Netherlands, 2009.

[9] R.S. Mans1, M.H. Schonenberg1, M. Song1, W.M.P. van der Aalst1, and P.J.M. Bakker2, Application of Process Mining in Healthcare - A Case Study in a Dutch Hospital, A. Fred, J. Filipe, and H. Gamboa (Eds.): BIOSTEC 2008, CCIS 25, pp. 425-438, 2008., springer 2008.

[10] Emir M. BahsiEmrah CeyhanTevfik Kosar, Conditional Workflow Management: A Survey and Analysis,Dblp, Scientific Programming 15(4):283-297, 2007

[11] A. Meidan n , J.A. García-García, M.J. Escalona, I. Ramos, A survey on business processes management suites,ACM ,2017

[12] Haoyan Wu 1, Zhijie Li 1, Brian King 1, Zina Ben Miled, ID, John Wassick 2 and Jeffrey Tazelaar, A Distributed Ledger for Supply Chain Physical Distribution Visibility, Information 2017.

[13] Zibin Z., ,Shaoan, X., Xiangping, C., and Huaimin, W., Blockchain Challenges and Opportunities: A Survey, Int. J. Web and Grid Services 1,2016

[14] Nakamoto, S, Bitcoin: Apeer-to-Peer Electronic Cash System. Available at: http://www.cryptovest.co.uk/resources/, Accessed at 2017.

[15] Tien Tuan Anh Dinh, Rui Liu, Meihui Zhang*, Untangling Blockchain: A Data Processing View of Blockchain Systems, 1041-4347 (c) 2017 IEEE. Translations and content mining are permitted for academic research only. Personal use is also permitted, but republication/redistribution requires IEEE permission. See

[16] Krystsina S., ,Adoptation of blockchain technologyu in supplychain and logistics, Bachelor of Business Logistics, 2017.

[17] Zhen, H., Zehua, W., Wei, C., and Victor, C.M., Lenug, BlockchainEmpowered Fair Computational Resource Sharing System in the D2D Network, Future Internet2017,9, 85; doi:10.3390/fi9040085

[18] DTCC. (2016). Embracing Disruption - Tapping the Potential of Distributed Ledgers to Improve the Post-Trade Landscape, 41 Deposit Trust \& Clearing Corporation. Available at: http://www.dtcc.com/ , Accessed at. 2017.

[19] Xiwei, X., Cesare, P., Liming, Z., Vincent, G., Alexander, P., AnBinh, T., Shiping, C., The Blockchain as a Software Connector, Software Architecture (WICSA), 2016 13th Working IEEE/IFIP Conference on, 2016.

[20] Michele, R., Floriano, S., Saverio, I., Giovanna, C., Giuseppe, L., Filippo, G., Semantic-enhanced blockchain technology for smartcities and communities, OWL 2 Web Ontology Language Document Overview (2nd Ed.), W3C Recommendation, 2012

[21] Tien Tuan, A.D.,, Ji, W., Gang, C., Rui, L., Beng, C.O, Kian-Lee, T. , BLOCKBENCH: A Framework for Analyzing Bitfury group ,Digital Assets on Public Blockchains, 2016 Private Blockchains, SIGMOD'17, Chicago, USA 2017.

[22] Zibin, Z., Shaoan, X., Hongning, D., Xiangping, C., and Huaimin, W., An Overview of Blockchain Technology: Architecture, Consensus, and Future Trends, IEEE 6th International Congress on Big Data, 2017.

[23] Christopher, K. F., \& Mariusz, N., From Institutions to Code: Towards Automated Generation of Smart Contracts, Foundations and Applications of Self* Systems, IEEE International Workshops on,2016
[24] Tiziana, c., From Bitcoin to The Internet of Things: The Role of The Blockchain, Annali della facoltà giuridica dell'università di camerino n. $6 / 2017$

[25] Raja, J., \& Praveen, G., Blockchain for IoT Security and Privacy: The Case Study of a Smart Home, Conference: IEEE Percom workshop on security privacy and trust in the internet of thing, 2017

[26] Michael, C., Nachiappan, S.V., and Vignesh, K., Blockchain Technology, Sutardja Center for Entrepreneurship \& Technology , Technical Report 2015.

[27] Florian, K., et al., Challenges and opportunities of digital information at the intersection of Big Data Analytics and Supply Chain Management, International Journal of Operations \& Production Management, Volume 37, Issue 1, 2015.

[28] Michael, R., \& Jan, V.B., The six core elements of business process management. In Handbook on Business Process Management 1. Springer, 105-122, 2015.

[29] Asaph, A., Ariel, E., Thiago, V., and Andrew, L., MedRec: Using Blockchain for Medical Data Access and Permission Management, 2nd International Conference on Open and Big Data, 2016.

[30] Richard, H., Vishal, S.B., Yi-Min, C., Alin, D., Fenno, F., Terry, H., and Victor, V., Towards a Shared Ledger Business Collaboration Language Based on Data-Aware Processes,Q.Z. Sheng et al. (Eds.): ICSOC 2016, LNCS 9936, pp. 18-36,Springer International Publishing Switzerland, 2016.

[31] Steve, H., Rituparna, B., Martin, W., Natalia, B., Internet of Things, Blockchain and Shared Economy Applications, Procedia Computer Science, Volume 98, 2016.

[32] Marko, V., Holger, S., Oliver, S., Bernhard, M., Volker, M., Albert, M., and Tobias, K., An Approach to Optimize Data Processing in Business Processes, VLDB Endowment, ACM 978-1-59593-649-3/07/09, 2007.

[33] Maria, L., \& Stefanie, R., A systematic review on security in ProcessAware Information Systems - Constitution, challenges, and future directions, Information and Software Technology 56, pages 273-293, 2014.

[34] Marlon, D., Marcello, L. R., Jan, M., and Hajo, A. R., Fundamentals of Business Process Management. Second Edition. Springer, 2018.

[35] Jan, v. B. \& Theresa, S., Culture in business process management: a literature review. Business Process Management Journal 17, pages 357378, 2011.

[36] Ye, G. \& Chen, L., Blockchain application and outlook in the banking industry. Financial Innovation 2, 1, 2016.

[37] Gregor, T., Standardization in technology-based markets. Research policy 29, 4, pages 587-602, 2000.

[38] Hyerim, B., Sanghyup, L., and kyeong, M., Planning of business process execution in Business Process Management environments, Information Sciences, Volume 268, Pages 357-369, 2014.

[39] Gareth, W. P., \& Guy, V., Overview of Emerging Blockchain Architectures and Platforms for Electronic Trading Exchanges. 2016.

[40] Ingo, W., Xiwei, X., Regis, R., Guido, G., Alexander, P., and Jan, M., Untrusted Business Process Monitoring and Execution Using Blockchain. In Business Process Management - 14th International Conference, BPM 2016, Rio de Janeiro, Brazil, 2016. Proceedings (Lecture Notes in Computer Science), Vol. 9850. Springer, 329-347. 